\title{
National Political Science Associations
}

Each spring $P S$ updates and publishes the names, officers, publications, and meeting dates of national political science associations around the world. Further additions and/or corrections for this list will be welcome as well as news items on activities and programs of national associations.

*These associations did not respond to our survey, and information is from previous editions of PS.

\begin{tabular}{|c|c|c|c|}
\hline Associations & Officers & Publications & Meetings \\
\hline $\begin{array}{l}\text { *AFRICA } \\
\text { African Political Science } \\
\text { Association } \\
\text { P.O. Box MP167, Mt. Pleasant } \\
\text { Harare, Zimbabwe }\end{array}$ & $\begin{array}{l}\text { President: Dani W. Nabudere } \\
\text { Vice President: H. Sharawi } \\
\text { Secretary General: L. Adele Jinadu }\end{array}$ & Newsletter, Ibbo Mandaza, Editor & Bi-annual Conference \\
\hline $\begin{array}{l}\text { ARGENTINA } \\
\text { *Asociacion Argentina de } \\
\text { Ciencia Politica } \\
\text { Avda. Alvear 1711, Piso } 3 \\
\text { (1014) Buenos Aires, Argentina } \\
\text { Phone: (541) 41-3066 }\end{array}$ & $\begin{array}{l}\text { President: Carlos A. Floria } \\
\text { Secretary: Daniel Funes de Rioja }\end{array}$ & $\begin{array}{l}\text { "Boletin de la Asociacion Argentina } \\
\text { de Ciencia Politica" }\end{array}$ & \\
\hline $\begin{array}{l}\text { *Argentine Society for Political } \\
\text { Analysis } \\
\text { Pueyrredon } 510,7 \mathrm{mo} \text {. piso, } \\
1032 \text { Buenos Aires }\end{array}$ & $\begin{array}{l}\text { President: Oscar Oszlak } \\
\text { Vice President: Abel Fleitas Ortiz de Rosas } \\
\text { Secretary: Waldino Suarez } \\
\text { Treasurer: Lilia Puig de Stubrin }\end{array}$ & $\begin{array}{l}\text { Sociedad Argentina de Analysis } \\
\text { Politico }\end{array}$ & \\
\hline $\begin{array}{l}\text { AUSTRALIA } \\
\text { Australasian Political Studies } \\
\text { Association (APSA) } \\
\text { Politics Dept., University } \\
\text { College, University of New } \\
\text { South Wales, ADFA, Canberra. } \\
\text { ACT, } 2600\end{array}$ & $\begin{array}{l}\text { President: Patrick Weller, Griffith } \\
\text { University } \\
\text { Vice President: Leslie Holmes } \\
\text { Treasurer: Brian Galligan } \\
\text { Secretary: Rhonda Moore }\end{array}$ & $\begin{array}{l}\text { Australian Journal of Political } \\
\text { Science, Ian McAllister, Editor } \\
\text { APSA Newsletter, David Gow, Dept. } \\
\text { of Government, University of } \\
\text { Queensland, Editor }\end{array}$ & $\begin{array}{l}\text { Annual Conference } \\
\text { July 17-19, 1991 } \\
\text { Griffith University } \\
\text { Brisbane, Queensland }\end{array}$ \\
\hline $\begin{array}{l}\text { *AUSTRIA } \\
\text { Austrian Political Science } \\
\text { Association } \\
\text { Stumpergasse 56, A-1060 } \\
\text { Vienna }\end{array}$ & $\begin{array}{l}\text { President: Emmerich Talos, University of } \\
\text { Vienna } \\
\text { Vice Presidents: Anselm Skuhra, University } \\
\text { of Salzburg; Barbara Wildauer, University } \\
\text { of Innsbruck } \\
\text { General Secretary: Karin Pintér, } \\
\text { Zukunftswerkstätte (Future atelier) } \\
\text { Treasurer: Silvia Michal-Misak, University } \\
\text { of Vienna }\end{array}$ & $\begin{array}{l}\text { Österreichische Zeitschrift für } \\
\text { Politikwissenschaft, Herbert } \\
\text { Gottweis, Ferdinant Karlhofer, } \\
\text { Wolfgang C. Müller, Margit Scherb, } \\
\text { Anselm Skuhra, Karl Ucakar, Editors }\end{array}$ & \\
\hline
\end{tabular}

*BELGIUM

Institut de Science Politique, c/o Fonds National de la Recherche Scientifique, Rue d'Egmont 5 B-1050, Brussels, Belgium

Politologisch Instituut (Flemish Political Science Association)
President: Ch. Goossenss, Université de

Liege

Vice President: A. Frognier, Université de Louvain

Directeur: A. Philippart, Université de

Libre de Bruxelles

Secretary: J. Leclercq, Université de Mons

Treasurer: M. P. Herremans, C.R.I.S.P.

Executive Committee: J. Beaufays, S.

Bernard, J. Buchmann, E. Cerexhe, P.

Dlaeys, F. Delperee, J. Gotovitch, G.

Gorley, X. Mabille, M. Molitor, F. Perin, M. Piraux, N. Vosswinkel

President: Hugo Van Hassel, University of Leuven
Res Publica: Belgian Journal of Political Science (articles in Dutch, 


\begin{tabular}{|c|c|c|}
\hline Associations & Officers & Publications \\
\hline $\begin{array}{l}\text { Egmontstraat, 11, B-1050, } \\
\text { Brussels, Belgium }\end{array}$ & $\begin{array}{l}\text { Vice Presidents: Maurice Boeynaems, Royal } \\
\text { Military Academy; Wilfried Dewachter, } \\
\text { University of Leuven; Jan Ceuleers, } \\
\text { University of Brussels } \\
\text { Secretary: Maurice Boeynaems } \\
\text { Treasurer: Yvan Vanden Berghe }\end{array}$ & $\begin{array}{l}\text { French, and English), Wilfried } \\
\text { Dewachter, Editor, E. Van Evenstraat } \\
\text { 2B B-3000, Leuven, Belgium }\end{array}$ \\
\hline $\begin{array}{l}\text { "BRAZIL } \\
\text { Sociedade Brasileira de } \\
\text { Instructao, Praca XV de } \\
\text { Novembro, 101 Rio de Janeiro, } \\
\text { Brazil 21-231-2946 }\end{array}$ & Administrator: Martha Pimenta De Moraes & $\begin{array}{l}\text { Revista de Ciencia Politica, Armando } \\
\text { de Oliveira Marinho, Editor }\end{array}$ \\
\hline $\begin{array}{l}\text { *BULGARIA } \\
\text { Bulgarian Political Science } \\
\text { Association } \\
100 \text {, Sofia, University of Sofia, } \\
15 \text { Rusky bul, Bulgarian } \\
\text { Political Life Research } \\
\text { Laboratory }\end{array}$ & Director: Mintcho Semov & $\begin{array}{l}\text { Annuaire de l'Université de Sofia } \\
\text { Laboratoir de recherches scientifiques } \\
\text { sur la vie politique des Bulgares }\end{array}$ \\
\hline $\begin{array}{l}\text { CANADA } \\
\text { Canadian Political Science } \\
\text { Association } \\
\text { \#205-1 Stewart St., Ottawa, } \\
\text { Ontario K1N 6H7 }\end{array}$ & $\begin{array}{l}\text { President: Vincent Lemieux, Université } \\
\text { Laval } \\
\text { President Elect: Peter H. Russell, University } \\
\text { of Toronto } \\
\text { Secretary-Treasurer: Simon McInnes, } \\
\text { Government of Canada } \\
\text { Administrator: Joan Pond }\end{array}$ & $\begin{array}{l}\text { Canadian Journal of Political Science, } \\
\text { Roger Gibbins, University of Calgary, } \\
\text { and Stéphane Dion, Université de } \\
\text { Montréal, Co-Editors } \\
\text { Bulletin, Gordon Mace, Université }\end{array}$ \\
\hline $\begin{array}{l}\text { Société québeçoise de science } \\
\text { politique } \\
\text { Department de science } \\
\text { politique, Université du Québec } \\
\text { à Montréal, C.P. 888, Succ. A, } \\
\text { Montreal, Quebec H3C 3P8 }\end{array}$ & $\begin{array}{l}\text { President: Duncan Cameron, Université } \\
\text { d’Ottawa } \\
\text { Secretary: Maurice Couture, Université du } \\
\text { Québec à Montréal }\end{array}$ & $\begin{array}{l}\text { Revue canadienne de science politique, } \\
\text { Roger Gibbins, University of Calgary, } \\
\text { and Stéphane Dion, Université de } \\
\text { Montréal, Co-Editors } \\
\text { Revue Politique, Carolle Simard, } \\
\text { l'Université du Québec à Montréal }\end{array}$ \\
\hline
\end{tabular}

CHILE
Asociacion Chilena de Ciencia
Politica
Catedral 1063 of. 34, Santiago
Phone: 6968379

CHINA
Chinese Association of
Political Science
c/o Chinese Academy of Social
Sciences, 5 Jiangoumennei
Ave., Beijing, People's
Republic of China 100732
Phone: $513-8263$

Phone: 513-8263

\section{"CZECHOSLOVAKIA}

Czechoslovak Political Science

Association

Narodni 18, 116 91, Praha 1

Phone: 203838
President: Gustavo Lagos

Secretary-General: Boris H. Yopo

President: Zhang Youyu
Executive Vice President: Li Shenzhi
Secretary-General: Wang Yunkun

Annuaire de l'Université de Sofia
Laboratoir de recherches scientifiques
sur la vie politique des Bulgares

\begin{tabular}{|c|c|c|c|}
\hline & $\begin{array}{l}\text { University of Bratislava, Safarikono n. } 6 \text {, } \\
\text { Bratislava } \\
\text { Secretary-General: Josef Skala, Institute of } \\
\text { Law, Narodni 18, Praha } 1\end{array}$ & & \\
\hline $\begin{array}{l}\text { DENMARK } \\
\text { Danish Political Science } \\
\text { Association } \\
\text { Department of Commercial } \\
\text { Law and Political Science, } \\
\text { Odense Universitet, Campusvej } \\
\text { 55-DK 5230, Odense M.- } \\
\text { Denmark } \\
\text { Phone: } 66158600 \\
\text { Telefax: } 66158428\end{array}$ & $\begin{array}{l}\text { President: Lars Bille, Dept. of Political } \\
\text { Science, Copenhagen University, } \\
\text { Rosenborggade 15, } 1130 \mathrm{KBH} \text {. K } \\
\text { Secretary: Kurt Klaudi Klausen, Dept. of } \\
\text { Commercial Law and Political Science, } \\
\text { Odense University } \\
\text { Treasurer: Peter Munk Christiansen, } \\
\text { Institute of Political Science, Aarhus } \\
\text { University } \\
\text { Executive Committee: Soren Risbjerg } \\
\text { Thomsen, Institute of Political Science, } \\
\text { Aarhus University; Niels-Henrik Topp, } \\
\text { Dept. of Political Science, Copenhagen } \\
\text { University }\end{array}$ & Newsletter (2-3 times a year) & Annual \\
\hline
\end{tabular}

President: Milan Matous, Institute of Marxism-Leninism, Valdestejnska 14, Praha 1

Vice Presidents: Josef Blahoz, Institute of

Law, Narodni 18, Praha 1; Jan Kvasnicka,

University of Bratislava, Safarikono n. 6,

ecretary-General: Josef Skala, Institute of
Bulletin of Czechoslovak Association of Political Science, Josef Biahoz, Editor 


\begin{tabular}{|c|c|c|c|}
\hline Associations & Officers & Publications & Meetings \\
\hline $\begin{array}{l}\text { FINLAND } \\
\text { Finnish Political Science } \\
\text { Association } \\
\text { University of Helsinki, } \\
\text { Aleksanterinkatu } 7,00100 \\
\text { Helsinki }\end{array}$ & $\begin{array}{l}\text { President: Tatu Vanhanen, University of } \\
\text { Tampere } \\
\text { Vice President: Dag Anckar, Abo Akademi } \\
\text { Secretary: Petty Heikkinen, University of } \\
\text { Helsinki } \\
\text { Treasurer: Nina Ramberg, University of } \\
\text { Helsinki }\end{array}$ & $\begin{array}{l}\text { Political Science in Finland (book } \\
\text { series) } \\
\text { Politiikka (The Main Journal of } \\
\text { Association), Kyosti Pekonen, Editor } \\
\text { Politiikan tutkimus \& yhteiskunta } \\
\text { (newsletter), Nina Ramberg, Editor }\end{array}$ & Annual \\
\hline $\begin{array}{l}\text { FEDERAL REPUBLIC OF } \\
\text { GERMANY } \\
\text { Deutsche Gesellschaft für } \\
\text { Politikwissenschaft e.V., c/o } \\
\text { Erhard Forndran, University of } \\
\text { Braunschweig, Wendenring 1-3, } \\
3300 \text { Braunschweig }\end{array}$ & $\begin{array}{l}\text { Chairman: Erhard Forndran, University of } \\
\text { Braunschweig } \\
\text { Vice Presidents: Peter Haungs, University } \\
\text { of Trier; Gesine Schwan, Free University } \\
\text { of Berlin; Peter Graf Kielmansegg, } \\
\text { University of Mannheim; Oscar Gabriel, } \\
\text { University of Mainz }\end{array}$ & $\begin{array}{l}\text { Zeitschrift für Politik/Jahresbände } \\
\text { Nomos Verl }\end{array}$ & $\begin{array}{l}\text { Annual } \\
\text { November }\end{array}$ \\
\hline $\begin{array}{l}\text { Deutsche Vereiningung für } \\
\text { Politische Wissenschaft } \\
\text { Institut für Politikwissenschaft, } \\
\text { Residenzschloss, } 6100 \\
\text { Darmstadt }\end{array}$ & $\begin{array}{l}\text { President: Bsate Kohler-Koch } \\
\text { Vice Presidents: Fran Lehner, Frieder } \\
\text { Nachold } \\
\text { Secretary: Brigitte Emig }\end{array}$ & $\begin{array}{l}\text { Politische Vierteljahresschrift } \\
\text { DVPW-Rundbrief ( } 2 \text { times a year) }\end{array}$ & $\begin{array}{l}\text { Triennial } \\
\text { October } 1991\end{array}$ \\
\hline $\begin{array}{l}\text { FRANCE } \\
\text { Asociation Française de Science } \\
\text { Politique } \\
224 \text { bd. St. Germain, } \\
75007 \text { Paaris } \\
\text { Phone: } 45-49-92-21\end{array}$ & $\begin{array}{l}\text { President: Georges Vedel } \\
\text { Secretary-General: Jean Luc Parodi } \\
\text { Assistant: Catherine Honnorat }\end{array}$ & $\begin{array}{l}\text { Revue française de science politique, } \\
\text { Jean-Luc Parodi }\end{array}$ & Triennial \\
\hline $\begin{array}{l}\text { *GERMAN DEMOCRATIC } \\
\text { REPUBLIC } \\
\text { National Association for } \\
\text { Political Science of the GDR } \\
1086 \text { Berlin (GDR), Otto- } \\
\text { Nuschke-Strasse, 10/11, } \\
\text { German Democratic Republic }\end{array}$ & $\begin{array}{l}\text { President: Karl-Heinz Roder, Institute for } \\
\text { Theory of State and Law, Academy of } \\
\text { Sciences of the GDR, Berlin } \\
\text { Vice-Presidents: Max Schmidt, Berlin; Rolf } \\
\text { Reißig, Berlin }\end{array}$ & $\begin{array}{l}\text { Staat und Recht, Academy for State } \\
\text { and Law, Potsdam-Babelsberg }\end{array}$ & Annual \\
\hline $\begin{array}{l}\text { *GREECE } \\
\text { Hellenic Political Science } \\
\text { Association } \\
\text { Omirou 19, Athens 10672, } \\
\text { Greece }\end{array}$ & $\begin{array}{l}\text { President: Paschalis M. Kitromides, } \\
\text { University of Athens } \\
\text { Secretary-General: Christos Lyrintzis, } \\
\text { University of Athens }\end{array}$ & $\begin{array}{l}\text { Occasional collective volumes on the } \\
\text { Greek political system and other } \\
\text { political issues }\end{array}$ & $\begin{array}{l}\text { Periodic professional } \\
\text { conferences on specific } \\
\text { political themes }\end{array}$ \\
\hline $\begin{array}{l}\text { *HUNGARY } \\
\text { Hungarian Political Science } \\
\text { Association } \\
\text { 1068 Budapest Benczur u. 33., } \\
\text { Hungary }\end{array}$ & $\begin{array}{l}\text { President: Peter Schmidt } \\
\text { Vice Presidents: Attila Agh, Mihaly Bihari, } \\
\text { Peter Hardi, Tamas Szentes } \\
\text { Secretary General: Gyorgy Szoboszlai }\end{array}$ & Year Book & $\begin{array}{l}\text { Annual Meeting } \\
\text { December }\end{array}$ \\
\hline $\begin{array}{l}\text { INDIA } \\
\text { Indian Political Science } \\
\text { Association } \\
\text { c/o K. P. Singh, General } \\
\text { Secretary and Treasurer, } \\
\text { Sheikhpura House, Opp. L.B.S. } \\
\text { Nagar Post Office, Patna } \\
800023 \text { (Bihar-India) }\end{array}$ & $\begin{array}{l}\text { President: Dr. K. M. Reddy, Osmania } \\
\text { University } \\
\text { Vice Presidents: Prof. A. M. Rajaselhariah, } \\
\text { Dharwad; Dr. V. M. Bachal, Poorva } \\
\text { University }\end{array}$ & $\begin{array}{l}\text { Indian Journal of Political Science, } \\
\text { R. Thandavan, Editor } \\
\text { Quarterly News Bulletin, K. P. Singh, } \\
\text { Editor }\end{array}$ & \\
\hline $\begin{array}{l}\text { IRELAND } \\
\text { Political Studies Association } \\
\text { of Ireland } \\
\text { Dept. of Political Science, } \\
\text { Trinity College, Dublin }\end{array}$ & $\begin{array}{l}\text { President: John Coakley, University of } \\
\text { Limerick } \\
\text { Vice-President: Jennifer Todd, University } \\
\text { College Dublin } \\
\text { Secretary: Michael Gallagher, Trinity } \\
\text { College, Dublin } \\
\text { Treasurer: Richard Jay, Queen's University, } \\
\text { Belfast }\end{array}$ & $\begin{array}{l}\text { Irish Political Studies, John Coakley } \\
\text { and Nicholas Rees, Editors } \\
\text { Bulletin, Michael Gallagher, Editor } \\
\text { Analyses of Irish general elections; } \\
\text { latest volume, How Ireland Voted } \\
1989\end{array}$ & $\begin{array}{l}\text { Annual Conference } \\
\text { Mid-October 199! } \\
\text { Belfast } \\
\text { One-day seminar to launch } \\
\text { Irish Political Studies, } \\
\text { Vol. 6, late June 1991 } \\
\text { Dublin }\end{array}$ \\
\hline $\begin{array}{l}\text { ISRAEL } \\
\text { Israel Political Science } \\
\text { Association } \\
\text { c/o Dept. of Political Studies, } \\
\text { Bar Ilan University, Ramat Gan } \\
\text { 52100, Israel }\end{array}$ & $\begin{array}{l}\text { President: Bernard Susser, Bar Ilan } \\
\text { University, Ramat Gan } \\
\text { Secretary: Asher Cohen, Bar Ilan }\end{array}$ & News Bulletin & $\begin{array}{l}\text { Annual Conference } \\
\text { May 28, } 1991\end{array}$ \\
\hline
\end{tabular}




\begin{tabular}{|c|c|c|c|}
\hline Associations & Officers & Publications & Meetings \\
\hline $\begin{array}{l}\text { ITALY } \\
\text { Società italiana di scienze } \\
\text { politica } \\
\text { Casella Postale } 216 \text { Torino } \\
\text { Centro, } 10100 \text { Torino, Italia }\end{array}$ & $\begin{array}{l}\text { President: Luigi Bonanate, University of } \\
\text { Turin } \\
\text { Association Secretary: Fabio Armao, } \\
\text { University of Turin }\end{array}$ & $\begin{array}{l}\text { Rivista Italiana di Scienza Politica, } \\
\text { Leonardo Morlino, Editor } \\
\text { Teoria politica, Luigi Bonanate, Editor } \\
\text { Rivista trimestrale di } \\
\text { scienza dell'amministrazione, Giorgio } \\
\text { Freddi, Editor }\end{array}$ & Annual Conference \\
\hline $\begin{array}{l}\text { JAPAN } \\
\text { The Japanese Political } \\
\text { Science Association } \\
\text { c/o Osaka City University, } \\
\text { Faculty of Law, 3-3-138 } \\
\text { Sugimoto, Sumiyoshi-ku, } \\
\text { Osaka 558, Japan }\end{array}$ & $\begin{array}{l}\text { President: Yasushi Yamaguchi } \\
\text { General Secretary and Treasurer: Toshio } \\
\text { Kamo }\end{array}$ & $\begin{array}{l}\text { The Annual of JPSA, Editorial Board } \\
\text { of JPSA }\end{array}$ & Annual Conference \\
\hline $\begin{array}{l}\text { KOREA } \\
\text { The Korean Political Science } \\
\text { Association } \\
\text { 304-28 Sachick-Dong, Chongro- } \\
\text { Gu, Seoul, 110-054, Korea }\end{array}$ & $\begin{array}{l}\text { President: Min Kang, Dankuk University } \\
\text { Vice-Presidents: Soong-Hoom Kil, Seoul } \\
\text { National University; Jin-Whan Thak, } \\
\text { Chunbook University } \\
\text { Secretary Treasurer: Myungsoon Shin, } \\
\text { Yonsei University } \\
\text { Research Director: Jang-Jip Choi, Korea } \\
\text { University }\end{array}$ & $\begin{array}{l}\text { The Korean Political Science Review } \\
\text { (biannually), Hong-Woo Kim, Seoul } \\
\text { National University, Editor } \\
\text { KPSA Newsletter (quarterly), Hong- } \\
\text { Kyoo Park, The Institute of Foreign } \\
\text { Affairs and National Security, Editor }\end{array}$ & $\begin{array}{l}\text { The Second World } \\
\text { Conference on Korean } \\
\text { Political Studies } \\
\text { September } \\
\text { Annual Conference } \\
\text { December }\end{array}$ \\
\hline $\begin{array}{l}\text { "MEXICO } \\
\text { Asociacion Mexicana de } \\
\text { Ciencia Politica } \\
\text { Calle de Asuncion n }{ }^{\circ} 15 \text { casa } 8 \text {, } \\
\text { Condominio "Villa Fontana" } \\
\text { San Jeronimo Lidice, Deleg. } \\
\text { Magdalena-Contreras } 10200, \\
\text { Mexico, C.F. }\end{array}$ & $\begin{array}{l}\text { Presidente: Renique Gonzalez Pedrero } \\
\text { Vice Presidents: Raul Bejar Navarro, Jorge } \\
\text { Carpizo, Andres Caso Lombardo, Hugo } \\
\text { Castro Aranda, Raul Cerdiel Reyes, Julio } \\
\text { Reyna, Leopoldo Solis, Abelardo Villegas, } \\
\text { Francisco Casanova Alvarez, Julieta } \\
\text { Guevra Bautista } \\
\text { Secretario General: Roberto Salcedo } \\
\text { Aquino } \\
\text { Tesorero: Jose Salgado } \\
\text { Secretaria Tecnica: Martha Hernandez }\end{array}$ & & \\
\hline $\begin{array}{l}\text { "NETHERLANDS } \\
\text { Dutch Political Science } \\
\text { Association } \\
\text { Twente University/BSK, Post- } \\
\text { box } 217,7500 \text { AE Enschede, } \\
\text { Holland } \\
\text { Phone: } 053-893201\end{array}$ & $\begin{array}{l}\text { President: A. Hoogerwerf, Twente } \\
\text { University } \\
\text { Secretary-Treasurer: K. R. D. Lulofs, } \\
\text { Twente University }\end{array}$ & $\begin{array}{l}\text { Acta Politica, K. Koch, Editor. } \\
\text { Business Correspondence: Boom } \\
\text { Publishing Cy., P.O. Box 58, } \\
\text { Meppel. Editorial Correspondence: } \\
\text { Faculteit der Sociale Wetenschappen, } \\
\text { Erasmus Universiteit, P.O. Box 1738, } \\
3000 \text { D.R. Rutterdam }\end{array}$ & $\begin{array}{l}\text { Conferences in May and } \\
\text { and November }\end{array}$ \\
\hline
\end{tabular}

\begin{tabular}{lll}
\hline NEW ZEALAND & President: Ramesh Thakur & Annual Conference \\
New Zealand Political Studies & Secretary: Chris Rudd & \\
Association & Treasurer: Stuart Greif & \\
Dept. of Political Studies, & & \\
University of Otago, P.O. Box & & \\
56, Dunedin, New Zealand & & \\
\hline
\end{tabular}

*NIGERIA President: Claude Ake

Nigerian Political Science

Association

c/o School of Political Sciences,

University of Port Harcourt,

P.M.B. 53232A, Port Harcourt, Nigeria

\section{*NORWAY}

Norwegian Political Science

Association

Postboks 8237, Hammersborg

0129 OSLO 1, Norway
Turin

Association Secretary: Fabio Armao,

University of Turin

Secretary Treasurer: Myungsoon Shin,

Research Director: Jang-Jip Choi, Korea

Presidente: Renique Gonzalez Pedrero

Castro Aranda, Raul Cerdiel Reyes, Julio

Reyna, Leopoldo Solis, Abelardo Villegas,

Guevra Bautista

Aquino

: Jose Salgado

President: A. Hoogerwerf, Twente

(

. Lulofs

President: Claude Ake
Kyoo Park, The Institute of Foreign

Affairs and National Security, Edito
Political Studies

tember

Anual Conference

December 


\begin{tabular}{lc} 
Associations & Officers \\
\hline PHILIPPINES & President: Carmencita T. Aguilar, \\
Philippine Political Science & University of the Philippines \\
Association & Vice-President: Socorro L. Reyes, De La \\
c/o Philippine Social Science & Salle University \\
Center, U.P., P.O. Box 205, & Secretary/Editor of PPSI: Olivia C. Caoili, \\
Diliman, Quezon City 3004, & University of the Philippines \\
Philippines & Treasurer: Shirley Advincula, Ateneo \\
& University \\
& PRO: Francisco Soriano, Lyceum \\
& Business Manager: Alex Brilliantes, \\
& University of the Philippines \\
& Auditor: Fe Sumilong, Pamantasan Ng \\
& Lungsod Ng Maynila (City of Manila \\
& University)
\end{tabular}

Publications

Philippine Political Science Journal, Olivia C. Caoili, University of the Philippines, Editor
Meetings

Annual Convention November 8-9, 1991 Phil. Social Science Center, Quezon City

\begin{tabular}{|c|c|c|c|}
\hline $\begin{array}{l}\text { *POLAND } \\
\text { Polish Political Science } \\
\text { Association } \\
\text { ul. Bednarska } 2 / 400-310 \text {, } \\
\text { Warszawa, Poland }\end{array}$ & $\begin{array}{l}\text { President: Henryk Groszyk, Uniwersytet } \\
\text { im. Marii Curie-Sklodowskiej, Lublin } \\
\text { 1st Vice President: Krzysztof Palecki, } \\
\text { Instytut Nauk Politycznych Uniwersytet } \\
\text { Jagiellonski, Krakow } \\
\text { Secretary-General: Karol B. Janowski, } \\
\text { Instytut ds. Mlodziezy KC PZPR } \\
\text { Warszawa }\end{array}$ & $\begin{array}{l}\text { Polish Political Yearbook, Longin } \\
\text { Pastusiak, Editor } \\
\text { Studia Nauk Politycznych, Artur } \\
\text { Bodnar, Editor } \\
\text { Edukacja Polityczna, Czeslaw } \\
\text { Mojsiewicz, Editor }\end{array}$ & \\
\hline $\begin{array}{l}\text { *ROUMANIA } \\
\text { Soseana Kiseleff, No. } 49 \\
\text { Bucarest } 1 \text {, cod. } 7000\end{array}$ & $\begin{array}{l}\text { President: George Macovescu, Université } \\
\text { de Bucarest, 1, cod. } 7000\end{array}$ & $\begin{array}{l}\text { Vittorul Social: Association romaine } \\
\text { des sciences politiques et Academia } \\
\text { des sciences sociales et politiques, } \\
11 \text { rue Onesti, Bucarest 1, cod. } 7000 \\
\text { Politikon, Michael Sinclair, Editor }\end{array}$ & \\
\hline $\begin{array}{l}\text { SOUTH AFRICA } \\
\text { Political Science Association } \\
\text { of South Africa } \\
\text { P.O. Box 1041, Florida, } 1710\end{array}$ & $\begin{array}{l}\text { President: Deon GeldenHuys } \\
\text { Vice President: Mervyn Frost } \\
\text { Secretary-Treasurer: Albert Venter }\end{array}$ & $\begin{array}{l}\text { Politikon, Mervyn Frost and Douglas } \\
\text { Irvine, Editors }\end{array}$ & October 1991 \\
\hline $\begin{array}{l}\text { *SPAIN } \\
\text { Spanish Political Science } \\
\text { Association } \\
\text { c/o Centro de Estudios } \\
\text { Constitucionales, Plaza de la } \\
\text { Marina Española, 9, } 28013 \\
\text { Madrid }\end{array}$ & $\begin{array}{l}\text { President: Manuel Jimenez de Parga, } \\
\text { Facultad de Derecho, Universidad } \\
\text { Complutense, Madrid } \\
\text { Secretary-General: Juan Jose Solozabal, } \\
\text { Facultad de Derecho, Universidad } \\
\text { Complutense, Madrid } \\
\text { Treasurer: Luis Aguiar Luque }\end{array}$ & & \\
\hline $\begin{array}{l}\text { SWEDEN } \\
\text { Swedish Political Science } \\
\text { Association } \\
\text { c/o Jon Pierre, Dept. of } \\
\text { Political Science, University of } \\
\text { Gothenburg, Sprängkullsgatan } \\
\text { 19, S-411 } 23 \text { Gothenburg, } \\
\text { Sweden }\end{array}$ & $\begin{array}{l}\text { President: Bo Särlvik, University of } \\
\text { Göteborg } \\
\text { Secretary: Jon Pierre, University } \\
\text { Göteborg }\end{array}$ & $\begin{array}{l}\text { Statsvetenskaplig Tidskrift, Lennart } \\
\text { Lundquist, Dept. of Political Science, } \\
\text { University of Lund, Box 52, S-22100 } \\
\text { Lund } \\
\text { Politologen (for members), c/o Jörgen } \\
\text { Hermansson, Dept. of Government, } \\
\text { University of Uppsala, Skytteanum, } \\
\text { Box 514, S-751 20 Uppsala, Sweden }\end{array}$ & \\
\hline $\begin{array}{l}\text { SWITZERLAND } \\
\text { Swiss Political Science } \\
\text { Association } \\
\text { Department of Political Science, } \\
\text { University of Geneva, } \\
7 \text {, route de Drize, } \\
1227 \text { Carouge-Geneva }\end{array}$ & $\begin{array}{l}\text { President: Pierre Allan, University of } \\
\text { Geneva } \\
\text { Vice President: Jürg Gabriel, University of } \\
\text { St. Gallen } \\
\text { Treasurer: Pascal Mahon } \\
\text { Secretary: Stephan Kux, University of } \\
\text { Zurich } \\
\text { Members: Urs Altermatt, Thomas Bernauer, } \\
\text { Roger Blum, Jean F. Freymond, Ulrich } \\
\text { Klöti, Peter Knoepfel, Hans Peter Kriesi, } \\
\text { Wolf Linder, Urs Luterbacher, Kurt } \\
\text { Nüssli, Ioannis Papadopoulos, Gerhard } \\
\text { Schmid, Daniel Seiler, Paolo Urio, } \\
\text { Ernest Wiebel }\end{array}$ & $\begin{array}{l}\text { Annuaire Suisse de Science Politique } \\
\text { Année Politique Suisse, Hans Peter } \\
\text { Kriesi, University of Geneva, Editor }\end{array}$ & Nov. of each year \\
\hline $\begin{array}{l}\text { *TAIWAN } \\
\text { Chinese Association of } \\
\text { Political Science } \\
102 \text { Kuang Fu South Road } \\
\text { (10th Floor), Taipei, Taiwan } 105 \\
\text { Phone: (02) } 772-0551\end{array}$ & $\begin{array}{l}\text { President: Fei-Lung Lui } \\
\text { Secretary-General: Jiun Han Tsao }\end{array}$ & $\begin{array}{l}\text { Annals of Political Science, Cheng } \\
\text { Wan Tsai, Editor }\end{array}$ & \\
\hline
\end{tabular}




\begin{tabular}{l} 
Associations \\
\hline *TURKEY \\
Turkish Political Science \\
Association \\
Faculty of Law, University \\
of Ankara, Cebeci-Ankara \\
Phone: 1275261 \\
*UNION OF SOVIET \\
SOCIALIST REPUBLICS \\
Soviet Political Science \\
Association \\
USSR Academy of Sciences, \\
119841 Moscow G-19, Frunze \\
str., 10, SPSA \\
Tel. 2918721
\end{tabular}

Officers

President: Ergun Ozbudun

Secretary-General: Metin Heper

Treasurer: Sina Aksin

Faculty of Law, University

Treasurer: Sina Aksin

President: Georgii Shakhnazarov

First Vice President: Mikhail Piskotin

Vice Presidents: Fiodor Burlatskii, Vladimir

Tumanov, Institute of State and Law;

Michael Marchenko, Moscow State

University

Secretary: Sergei Deitsev, SPSA, USSR

Academy of Sciences

Treasurer: Alisa Tiain

\section{UNITED KINGDOM}

Political Studies Association

of the United Kingdom

Dept. of Public Administration,

University of Ulster, Derry,

BT48 7JL, N. Ireland

Phone: (0504) 265621

FAX: (0504) 262761
Chairman: Michael Goldsmith, University of Salfory

Honorary Secretary: Neil Collins,

University of Ulster

Honotary Treasurer: Jim Chandler Sheffield Polytechnic

Members: Ian Gordon, Kingston Polytechnic; David Morgan, University of Liverpool; David Denver, University of Lancaster; Pipa Norris, University of Edinburgh; Ursula Vogel, University of Manchester; Howard Elcock, Newcastle Polytechnic; Bill Miller, University of Glasgow; Andrew Reeve, University of Warwick; Vince Geoghegan, University of Belfast

UNITED STATES President: Theodore J. Lowi, Cornell

OF AMERICA

American Political Science Association

1527 New Hampshire Ave., NW, Washington, DC 20036

University

President-elect: James Q. Wilson, University of California, Los Angeles

Vice Presidents: William J. Daniels, Rochester Institute of Technology; Gerhard Loewenberg, University of Iowa; Kenneth Prewitt, Rockefeller Foundation Secretary: Charles Lipson, University of Chicago

Treasurer: David Brady, Stanford

University

Executive Director: Catherine E. Rudder

Program Chair: George H. Quester,

University of Maryland
Annual of the Soviet Political Science Association, Djangir Kerimov, Editor in Chief
February of each year
Political Studies, Jack Haywood, University of Hull, Editor

Politics, Andrew Taylor, Polytechnic

of Huddersfield, Editor

Newsletter, John Benyon, University

of Leicester

\section{VENEZUELA}

Asociacion Venezolana de Ciencia Politica

Calle 34 No. 13B-85, Doral Norte, PO Box 361, Maracaibo 4001-A
President: Hermann Petzold-Pernia,

Universidad del Zulia

Treasurer: Anton Zambrano, Universidad

del Zulia

\section{YUGOSLAVIA}

Union of Political Sciences

Associations of Yugoslavia 11000 Beograd, Proleterskih brigada 74, Yugoslavia
President: Zdravko Tomac, University of

Zagreb

Secretary: Vukasin Pavlovic, University of

Belgrade

Treasurer: Danka Firaunovic
American Political Science Review, Samuel C. Patterson, Ohio State University, Editor PS: Political Science \& Politics, Robert J-P., Hauck, APSA, Editor The Political Science Teacher, Sheilah Mann, APSA, Editor (See APSA publications list at back of PS.)
Annual

Aug. 29-Sept. 1, 1991

Washington, DC

Treasurer: Danka Firaunovic




\section{An Update of Regional and State Associations}

The following associations have updated their information since the March issue of PS.

\begin{tabular}{|c|c|c|c|}
\hline Associations & Officers & Publications & Meetings \\
\hline $\begin{array}{l}\text { Iowa Conference of } \\
\text { Political Scientists }\end{array}$ & $\begin{array}{l}\text { President: Dennis Goldford, Drake } \\
\text { University } \\
\text { Secretary-Treasurer: Bruce Nesmith, Coe } \\
\text { College }\end{array}$ & & $\begin{array}{l}\text { November 9, } 1991 \\
\text { Coe College } \\
\text { Cedar Rapids, IA }\end{array}$ \\
\hline $\begin{array}{l}\text { Mississippi Political } \\
\text { Science Association }\end{array}$ & $\begin{array}{l}\text { President: Stephen D. Shaffer, Mississippi } \\
\text { State University } \\
\text { Vice President: Elizabeth A. Sharpe, } \\
\text { Jackson State University } \\
\text { Secretary-Treasurer: Kate Greene, } \\
\text { University of Southern Mississippi }\end{array}$ & & Annual \\
\hline $\begin{array}{l}\text { New York State Political } \\
\text { Science Association }\end{array}$ & $\begin{array}{l}\text { President: Nancy E. McGlen, Niagara } \\
\text { University } \\
\text { Vice President and } 1992 \text { Program Chair: } \\
\text { Robert Heineman, Alfred University } \\
\text { Secretary-Treasurer: Leon Halpert, Siena } \\
\text { College }\end{array}$ & & \\
\hline $\begin{array}{l}\text { Ohio Association of Economists } \\
\text { and Political Scientists }\end{array}$ & $\begin{array}{l}\text { President: Carl Lieberman, University of } \\
\text { Akron } \\
\text { Vice President: Jean Sandver, Capital } \\
\text { University } \\
\text { Treasurer: W. Robert Gump, Miami } \\
\text { University } \\
\text { Secretary: Henry Rennie, University of } \\
\text { Toledo }\end{array}$ & $\begin{array}{l}\text { Ohio Journal of Economics and } \\
\text { Politics, James Sperling, University } \\
\text { of Akron, Political Science Editor; } \\
\text { Joseph C. Gallo, University of } \\
\text { Cincinnati, Economics Editor; and } \\
\text { Ernest Brass III, Capital University, } \\
\text { Managing Editor } \\
\text { Newsletter, Leo Navin, Bowling Green } \\
\text { University, Editor }\end{array}$ & $\begin{array}{l}\text { Annual } \\
\text { October } 18-19,1991 \\
\text { Capital University } \\
\text { Columbus, Ohio }\end{array}$ \\
\hline $\begin{array}{l}\text { Oklahoma Political } \\
\text { Science Association }\end{array}$ & $\begin{array}{l}\text { President: Harry A. Holloway, University } \\
\text { of Oklahoma } \\
\text { President-Elect/Vice President: Gary L. } \\
\text { Tompkins, Western Oklahoma State } \\
\text { University } \\
\text { Secretary-Treasurer: Edward Rolison, } \\
\text { Western Oklahoma State University }\end{array}$ & & \\
\hline
\end{tabular}

